factors have a compact product ${ }^{3}$ and the product of the remaining factors is metrisable. ${ }^{4}$

REMARK. In Theorem 4, the hypothesis that the factor spaces be metric cannot be much weakened. This is shown by an example of R. H. Sorgenfrey (see [4]), in which the product of a paracompact (and thus fully normal) space with itself is not even normal.

\title{
BIBLIOGRAPHY
}

1. P. Alexandroff and H. Hopf, Topologie I, Berlin, 1935.

2. J. Dieudonné, Une généralisation des espaces compacts, J. Math. Pures Appl. vol. 23 (1944) pp. 65-76.

3. C. Kuratowski, Topologie I, Warsaw 1933.

4. R. H. Sorgenfrey, On the topological product of paracompact spaces, Bull. Amer. Math. Soc. vol. 53 (1947) pp. 631, 632.

5. J. W. Tukey, Convergence and uniformity in general topology, Annals of Mathematics Studies, no. 2, Princeton, 1940.

Trinity College, University of Cambridge

${ }^{3}$ A theorem of Tychonoff; see, for example, [5, p. 75] for a simple proof.

4 See, for example, [3, p. 88].

\section{TRANSITIVITY AND EQUICONTINUITY ${ }^{1}$}

\section{W. H. GOTTSCHALK}

Let $X$ be a metric space with metric $\rho$ and let $G$ be a group of homeomorphisms on $X$. If $x \in X$ and $g \in G$, then $x g$ denotes the image of the point $x$ under the transformation $g$. If $x \in X$ and $F \subset G$, then $x F$ denotes $\bigcup_{o \in F} x g . G$ is said to be algebraically transitive provided that $x G=X$ for some $x \in X$ (and therefore for every $x \in X$ ). $G$ is said to be topologically transitive provided that $(x G)^{*}=X$ for some $x \in X$, where the star denotes the closure operator. $G$ is said to be equicontinuous provided that to each $\epsilon>0$ there corresponds $\delta>0$ such that $x, y \in X$ with $\rho(x, y)<\delta$ implies $\rho(x g, y g)<\epsilon(g \in G)$.

With respect to the following lemma compare [4].2

LEMMA. If $X$ is a complete separable metric space and also a multiplicative group, if the center of $X$ is dense in $X$ and if the function $x y$

Presented to the Society, December 31, 1947; received by the editors November 29, 1947.

1 Prepared under the sponsorship of the Office of Naval Research.

${ }^{2}$ Numbers in brackets refer to the bibliography at the end of the paper. 
$(x, y \in X)$ is continuous in $x$ for each fixed $y$, then the function $x y$ is continuous simultaneously in $x$ and $y$.

Proof. We first show that $x y$ is continuous in $y$ for each fixed $x$. Let $x_{0} \in X$ and let $\left\{x_{n}\right\}$ be a sequence of points in the center of $X$ such that $x_{n} \rightarrow x_{0}$. Now $y \in X$ implies $y x_{n}=x_{n} y \rightarrow x_{0} y$. Hence the sequence $\left\{y x_{n}\right\}$ of continuous functions of $y$ converges pointwise to the function $x_{0} y$ of $y$. Thus $x_{0} y$ is of Baire class 1 and has at least one point of continuity. (See [3, pp. 184, 189].) By right translation it follows that $x_{0} y$ is continuous in $y$ for all values of $y$.

Since $x y$ is continuous in $x$ and $y$ separately, $x y$ is of Baire class 1 in $(x, y)$. (See $[3$, p. 180].) Hence $x y$ has at least one point $(x, y)$ of continuity and by left and right translations we see that $x y$ is continuous in $(x, y)$ for all values of $(x, y)$.

THEOREM. If $X$ is a compact metric space, if $G$ is a topologically transitive abelian group of homeomorphisms on $X$ and if $H$ is the group of all homeomorphisms on $X$ which commute with every element of $G$, then the following statements are pairwise equivalent: (1) $H$ is algebraically transitive; (2) $H$ is equicontinuous; (3) $G$ is equicontinuous.

Proof. Assume $H$ is algebraically transitive. There exists $e \in X$ such that $(e G)^{*}=X$. If $x \in X, h \in H$ and $e h=e$, then there exists a sequence $\left\{g_{n}\right\}$ in $G$ such that $e g_{n} \rightarrow x$ whence $e g_{n}=e h g_{n}=e g_{n} h \rightarrow x h$ and $x h=x$. We conclude that for $x \in X$ there exists exactly one element of $H$, denoted $h_{x}$, such that $e h_{x}=x$. Define a product in $X$ as follows: If $x, y \in X$, then $x y=e h_{x} h_{y}$. It is readily verified that $X$ is a group such that every element of $e G$ commutes with every element of $X$, $e G$ is dense in $X$ and the function $x y$ is continuous in $x$ for each fixed $y$. By the lemma, $x y$ is continuous on $X \times X$. Since $X \times X$ is compact, $x y$ is uniformly continuous on $X \times X$. It follows that to each $\epsilon>0$ there corresponds $\delta>0$ such that $x, y \in X$ with $\rho(x, y)<\delta$ implies $\rho(x z, y z)<\epsilon(z \in X)$. This statement is just the statement of the equicontinuity of $H$. Thus we have shown that (1) implies (2).

Obviously (2) implies (3).

Assume $G$ is equicontinuous. Let $C$ denote the space of all continuous transformations of $X$ into $X$ supplied with the usual metric. Since $C$ is complete and $G$ is totally bounded (see [1]), $G^{*}$ in $C$ is compact. Choose $e \in X$ so that $(e G)^{*}=X$. Let $x \in X$ and let $\left\{g_{n}\right\}$ be a sequence in $G$ for which $e g_{n} \rightarrow x$. Select a subsequence $\left\{g_{n_{i}}\right\}$ of $\left\{g_{n}\right\}$ so that $g_{n_{i}} \rightarrow h \in G^{*}$ and $g_{n_{i}}^{-1} \rightarrow h_{0} \in G^{*}$. Now $y \in X$ implies $y=\left(y g_{n_{i}}\right) g_{n_{i}}^{-1}$ $\rightarrow(y h) h_{0}$ and $y=y h h_{0}$. Similarly $y=y h_{0} h(y \in X)$. Hence $h$ is a homeomorphism of $X$ onto $X$. Since also $h g=g h(g \in G)$, we conclude that 
$h \in H$. Furthermore $e h=x$. Thus $e H=X$ and $H$ is algebraically transitive. The proof is completed.

The following corollary solves a problem proposed by Hedlund [2, bottom p. 617].

CoRollary. Let a flow be defined on a compact metric space $X$ so that $X$ is a minimal orbit-closure. Then the flow is equicontinuous if and only if for every pair of points of $X$ there exists an orbit-preserving homeomorphism on $X$ transforming one of these points into the other.

The above corollary permits a rephrasing of a conjecture of G. D. Birkhoff [5, problems 2 and 3], namely: If a continuous flow on an $n$-dimensional manifold is pointwise almost periodic, then the flow is almost periodic (equicontinuous) on each orbit-closure. (See [1] for terms used.)

\section{BIBLIOGRAPHY}

1. W. H. Gottschalk, Almost periodicity, equi-continuity and total boundedness, Bull. Amer. Math. Soc. vol. 52 (1946) pp. 633-636.

2. G. A. Hedlund, Sturmian minimal sets, Amer. J. Math. vol. 66 (1944) pp. 605620.

3. C. Kuratowski, Topologie I.

4. Deane Montgomery, Continuity in topological groups, Bull. Amer. Math. Soc. vol. 42 (1936) pp. 879-882.

5. G. D. Birkhoff, Some unsolved problems of theoretical dynamics, Science vol. 94 (1941) pp. 598-600.

INSTITUTE FOR AdVANCED STUdy AND University of Pennsylvania 